Zur Reaktivität von $\mathrm{C}=\mathrm{N}$-Doppelbindungssystemen, $\mathrm{I}$

\title{
Eine Synthese des 1.4-Diphenyl-azetidin-2-on
}

\author{
The Reactivity of the $\mathrm{C}=\mathrm{N}$ Double Bond System, I \\ The Synthesis of 1,4-Diphenyl-azetidin-2-one \\ Erich Ziegler, Walter Ott und Michael Riegler \\ Institut für Organische Chemie der Universität Graz \\ (Z. Naturforsch. 29b, 677-678 [1974]; eingegangen am 16. Mai 1974) \\ $\mathrm{N}$-( $\alpha$-acetoxybenzyl)-acetanilide, 1,4-Diphenyl-azetidin-2-one
}

\begin{abstract}
The adduct from benzylidene aniline and acetic anhydride gives 1,4-diphenyl-azetidin-2one if heated at $210^{\circ} \mathrm{C}$ in diphenylmethane or diphenylsilane.
\end{abstract}

Im Laufe der Zeit sind, wie aus verschiedenen Monographien ${ }^{1-3} \mathrm{zu}$ entnehmen ist, zahlreiche Verfahren zur Herstellung von $\beta$-Lactamen ausgearbeitet worden. So können u.a. Benzalanilin und seine Derivate mit monosubstit. Malon ${ }^{4}$ - bzw. Cyanessigsäurechloriden ${ }^{5}$ zu $\beta$-Lactamen umgesetzt werden. Ferner vermag man Mono- und Dihalogenessigsäuren in Dimethylformamid mit Hilfe von $\mathrm{POCl}_{3}{ }^{6}$ direkt an Benzalanilin unter Bildung von Acetidinonen zu addieren.

Es läßt sich nun zeigen, daß das schon lange bekannte 1:1 Addukt? aus Benzalanilin und Essigsäureanhydrid beim Erhitzen in inerten Lösungsmitteln auf $210{ }^{\circ} \mathrm{C}$ zum 1.4-Diphenyl-acetidin-2-on. $(25 \%)$ reagiert. Dieses Verfahren erinnert an jenes von Bose, Kapur, Sharma und Mohas ${ }^{8}$, das die Umsetzung von Anilen mit gemischten Anhydriden in Gegenwart von Triäthylamin zum Gegenstand hat.

Der erwähnte 1:1-Komplex zwischen Benzalanilin und Acetanhydrid ist in seiner Struktur aber noch keineswegs sichergestellt. SNyder, BRIGHT und LORENZ ${ }^{9}$ bzw. BURGSTAHLER ${ }^{10}$ formulieren diese Verbindung als $\mathrm{N}$-( $\alpha$-Acetoxybenzyl)-acetanilid (1), während Passerini und Macenteleli ${ }^{11}$ der Ansicht sind, daß es sich bei diesem Addukt um ein ionisches Molekül von der Struktur 2 handelt.

Aufgrund von ${ }^{13} \mathrm{C}$ NMR-Messungen läßt sich aber beweisen, daß dem Addukt bei $20{ }^{\circ} \mathrm{C}$ die Struktur 1 zukommt. Für das im Anil doppelt gebundene C

Sonderdruckanforderungen an Prof. Dr. E. Ziegler, Institut für Organische Chemie der Universität, A-8010 Graz, Heinrichstr. 28, Österreich. findet man den Wert von $160,8 \mathrm{ppm}$, der sich nach Anlagerung von Acetanhydrid zu 79,0 ppm verschiebt. Diese Verschiebung um mehr als $80 \mathrm{ppm}$ in das typische Aliphatengebiet weist eindeutig auf eine $\mathrm{CH}-\mathrm{N}$-Einfachbindung in $\mathbf{1}$ hin. $\mathrm{Ob}$ bei höheren Temperaturen diesem Produkt ein teilweise ionischer Charakter (etwa vom Typ 2) zuzuschreiben ist, bleibt noch offen, manche Folgereaktionen sprechen aber dafür.

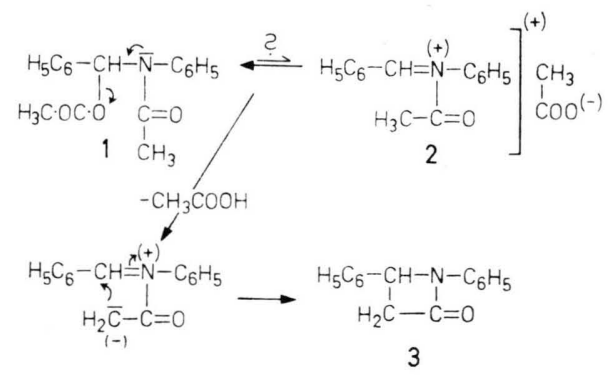

Sicher abzulehnen ist dagegen ein Strukturvorschlag von ANGel und DAY ${ }^{12}$, die meinen, daß eine der $\mathrm{CH}_{3}$-Gruppen des Acetanhydrids sich an die $\mathrm{C}=\mathrm{N}$-Gruppierung des Aniles unter Bildung des Essigsäure- $\beta$-anilino-dihydrozimtsäure-anhydrids anlagert.

ANGEL und DAY ${ }^{12}$ teilen ferner mit, daß beim trockenen Erhitzen des Adduktes durch 20 Min. auf $220{ }^{\circ} \mathrm{C}$ im $\mathrm{N}_{2}$-Strom ein quantitativer Zerfall desselben in Benzalanilin und Acetanhydrid stattfindet. In Lösung (z.B. Diphenylsilan, $210^{\circ} \mathrm{C}$ ) aber entsteht, wie wir beobachtet haben, das 1.4-Diphenylazetidin-2-on ${ }^{13}$ (3). In Analogie dazu soll auf 
Arbeiten von Yoshid ${ }^{13}$ verwiesen werden, wonach Schiffsche Basen des $\alpha$ - bzw. $\beta$-Phenylalaninesters mit Dichloressigsäure-anhydrid relativ gut zu $\beta$ Lactamen reagieren.

Entsprechend dem Benzalanilin lassen sich auch Benzyliden-o- bzw. - $p$-toluidin mit Essigsäureanhydrid zu analog aufgebauten Verbindungen zusammenlagern. Ferner ist das Addukt aus Benzalanilin und Propionsäureanhydrid leicht zugänglich. Diese Abkömmlinge erfahren beim Erhitzen in Lösungsmitteln weitgehende Zersetzung, die zu erwartenden $\beta$-Lactame bilden sich meist nur in geringer Menge bzw. gar nicht.

Von größerem Interesse ist wohl die Beobachtung, daß Addukte vom Typ 1 mit methylenaktiven Verbindungen in folgendem Sinne reagieren:

$$
\begin{aligned}
1+\mathrm{H}_{2} \mathrm{C}_{\mathrm{y}}^{\mathrm{x}} \rightarrow \mathrm{CH}_{3} \cdot \mathrm{COOH} & +\mathrm{C}_{6} \mathrm{H}_{5}-\mathrm{CH}=\mathrm{C}<_{\mathrm{y}}^{\mathrm{x}} \\
& + \text { Acetanilid }
\end{aligned}
$$

Diese „Olefinierungs-Reaktion“ ist auf alle Systeme (z.B. Oxime, Hydrazone etc.) übertragbar, die im Molekül eine isolierte $\mathrm{C}=\mathrm{N}$-Doppelbindung aufweisen. Bei der Einwirkung von $\mathrm{H}_{2} \mathrm{~S}$ entstehen naturgemäß Thioketone und bei der von Aminen wird der ursprünglich eingebaute Aminbaustein durch den neueintretenden völlig verdrängt. Über diese Beobachtungen soll demnächst berichtet werden.

\section{Experimentelles}

Die IR-Spektren wurden mit einem PerkinElmer-Spektrophotometer 421, die HNMR-Spektren mit einem Varian-HA-100-D-Kernresonanzgerät aufgenommen.

\section{$N$-( $\alpha$-Acetoxybenzyl)-acetanilid ${ }^{9,10}(\mathbf{1})$}

$18,1 \mathrm{~g}(0,1 \mathrm{Mol})$ Benzalanilin und 10,2 ml (0,1 Mol) Acetanhydrid werden nach Zugabe von 2 ml Eisessig 1 Stde. unter Rückfluß erhitzt. Nach Anreiben mit Petroläther und Umkristallisieren aus Ligroin erhält man $21 \mathrm{~g}(74 \%)$ an 1, Schmp. $130-131{ }^{\circ} \mathrm{C}$.

1 J. C. Shenhan und E. J. Corey, Org. Reactions 9, 388 [1957].

2 Th. Wieland, Houben-Weyl, Methoden der organischen Chemie, vierte Auflage, Bd. XI/2, S. 518, Georg Thieme Verlag, Stuttgart 1958.

${ }^{3}$ A. K. Mukerjee und R. C. Srivastava, Organic Synthesis 6, 327 [1973].

${ }^{4}$ E. Ziegler und G. Kleineberg, Monatsh. Chem. 96, 130 [1966].

${ }^{5}$ E. Ziegler und Th. Wimmer, Chem. Ber. 99, 130 [1966].

${ }^{6}$ E. Ziegler, Th. Wimmer und H. Mittelbach, Monatsh. Chem. 99, 2128 [1968].

7 Beilsteins Handbuch d. Org. Chemie, zweites Er-

\section{$N$-(a-Acetoxybenzyl)-4-methylacetanilid}

$19,5 \mathrm{~g}(0,1 \mathrm{Mol})$ Benzyliden- $p$-toluidin werden mit $10,2 \mathrm{ml}(0,1 \mathrm{Mol}) \mathrm{Ac}_{2} \mathrm{O}$ und $2 \mathrm{ml}$ Eisessig $2 \mathrm{Stdn}$. auf $160{ }^{\circ} \mathrm{C}$ erhitzt. Nach 24 -stg. Stehen bei $0{ }^{\circ} \mathrm{C}$ kristallisiert man aus Cyclohexan. $14 \mathrm{~g}$ farbloser Prismen $(47,1 \%)$ vom Schmp. 95-96 ${ }^{\circ} \mathrm{C}$.

$\mathrm{C}_{18} \mathrm{H}_{19} \mathrm{~N}_{2} \mathrm{O}_{3} \quad(297,4)$

Ber. N 4,71 ,

Gef. N 4,88.

\section{$N$-(a-Acetoxybenzyl)-2 methylacetanilid}

Analog aus 19,5 g Benzyliden-o-toluidin. Aus Cyclohexan $13 \mathrm{~g}$ Kristalle $(44 \%$ ) vom Schmp. 81 bis $82^{\circ} \mathrm{C}$.

$\mathrm{C}_{18} \mathrm{H}_{19} \mathrm{~N}_{2} \mathrm{O}_{3} \quad(297,4)$

Ber. N 4,71 ,

Gef. N 4,73 .

\section{$N$-(a-Propionyloxybenzyl)-anilinpropionat}

$18,1 \mathrm{~g}(0,1 \mathrm{Mol})$ Benzalanilin werden mit $13 \mathrm{~g}$ (0,1 Mol) Propionsäureanhydrid und $2 \mathrm{ml}$ Propionsäure 12-14 Stdn. auf $100^{\circ} \mathrm{C}$ erhitzt. Nach Reiben der Lösung mit dem Glasstab kommt es zur Kristallisation. Aus Cyclohexan farblose Kristalle vom Schmp. $121{ }^{\circ} \mathrm{C}$. Ausb. 11,2 g $(68,7 \%)$.

\section{4-Diphenyl-azetidin-2-on ${ }^{14}(3)$}

$2,83 \mathrm{~g} 1(0,01 \mathrm{Mol})$ werden in $2 \mathrm{ml}$ Diphenylsilan 90 Min. auf $210^{\circ} \mathrm{C}$ erhitzt, wobei Essigsäure aus dem Steigrohr entweicht. Nach Versetzen des Reaktionsgutes mit $8 \mathrm{ml}$ Äther-Äthanol (1:1) fällt bei $0{ }^{\circ} \mathrm{C} 3$ nach einigen Stunden in farblosen Nadeln an. Aus Methanol erh ältman 0,55 g $(25 \%) 3$ vom Schmp. $152{ }^{\circ} \mathrm{C}$.

IR (in $\mathrm{KBr}): 1740 \mathrm{~cm}^{-1}(\mathrm{C}=\mathrm{O})$. NMR $\left(\mathrm{inCDCl}_{3}\right.$, $100 \mathrm{~Hz})$ :

$\mathrm{CH}_{2} \delta=2,9$ und $3,6 \mathrm{ppm}, J 15^{\mathrm{c}} / \mathrm{s}, 5 \mathrm{c} / \mathrm{s}$ und $2 \mathrm{c} / \mathrm{s}$, $\mathrm{CH}^{2} \delta=5,0 \mathrm{ppm}, J 5^{\mathrm{c}} / \mathrm{s}$ und $2 \mathrm{c} / \mathrm{s}$,

Aromat $\delta=7,3 \mathrm{ppm}$.

$\mathrm{C}_{15} \mathrm{H}_{13} \mathrm{NO}(223,3)$

Ber. N 6,27,

Gef. N 6,50 .

Für die Unterstützung dieser Arbeit danken wir der Ciba-Geigy AG, Basel.

gänzungswerk, Bd. XII, S. 144, Springer-Verlag, Berlin, Göttingen, Heidelberg 1950.

8 A. K. Bose, J. C. Kapur, S. D. Sharma und M. S. MoHas, Tetrahedron Lett. 1973, 2319.

${ }^{9}$ H. R. SNyder, D. B. Bright und J. C. Lorenz, J. Amer. Chem. Soc. 73, 1836 [1951].

10 A. W. Burgstahler, J. Amer. Chem. Soc. 73, 3021 [1951].

11 M. Passerini und M. P. Macentelli, Gazz. Chim. Ital. 58, 64 [1928].

12 H. S. Axger und A. R. Day, J. Amer. Chem. Soc. 72, $3874[1950]$.

13 Norio Yoshida, C 1965, 51, 16221 u. 16223.

${ }^{14}$ H. Staudinger, Ber. Deut. Chem. Ges. 50, 103 [1917]. 